\title{
Erratum
}

\section{Dynamics control of semantic processes in a hierarchical associative memory}

\section{Bo Cartling}

Department of Theoretical Physics, The Royal Institute of Technology, S-100 44 Stockholm, Sweden

Biol. Cybern. 74, 63 - 71 (1996)

Due to a typing error $g_{k}^{i}\left(i_{k}^{i}(t)\right)$ in equation (3) should be replaced by $g_{k}^{p}\left(i_{k}^{p}(t), c_{k}^{p}(t)\right)$.

The activity of a pyramidal unit $s_{j}^{p}(t)$ in equations (9) and (12) is equal to $g_{j}^{p}\left(i_{j}^{p}(t), c_{j}^{p}(t)\right)$. 